\title{
A Complete Framework of Radar Pulse Detection and Modulation Classification for Cognitive EW
}

\author{
Ersin $\operatorname{Yar}^{\dagger \ddagger}$, Mehmet Burak Kocamis ${ }^{\dagger}$, Adnan Orduyilmaz ${ }^{\dagger *}$, Mahmut Serin ${ }^{\dagger}$, Murat Efe* \\ ${ }^{\dagger}$ TUBITAK BILGEM ILTAREN, Ankara, Turkey \\ * Department of Electrical and Electronics Engineering, Ankara University, Ankara, Turkey \\ ${ }^{\ddagger}$ Department of Electrical and Electronics Engineering, Bilkent University, Ankara, Turkey \\ E-mail:\{ersin.yar,burak.kocamis,adnan.orduyilmaz,mahmut.serin@tubitak.gov.tr\} \\ E-mail:\{murat.efe@eng.ankara.edu.tr\}
}

\begin{abstract}
In this paper, we consider automatic radar pulse detection and intra-pulse modulation classification for cognitive electronic warfare applications. In this manner, we introduce an end-to-end framework for detection and classification of radar pulses. Our approach is complete, i.e., we provide raw radar signal at the input side and produce categorical output at the output. We use short time Fourier transform to obtain timefrequency image of the signal. Hough transform is used to detect pulses in time-frequency images and pulses are represented with a single line. Then, convolutional neural networks are used for pulse classification. In experiments, we provide classification results at different SNR levels.
\end{abstract}

Index Terms-Cognitive EW, pulse detection, intra-pulse modulation classification, convolutional neural networks

\section{INTRODUCTION}

Modern radars have the ability of changing their waveform and modulation type in a quick and agile way even from pulse to pulse. Moreover, MOP and antenna scan type classification are important in terms of obtaining critical parameters to overcome uncertainties for deinterleaving since other parameters in modern radar such as frequency and pulse width are not discriminative. Automatic modulation on pulse (MOP) extraction is necessary in order to detect these changes. Hence, cognitive electronic warfare (EW) provides rapid solutions in a detailed way. In [1], automatic antenna scan type classification is investigated.

Furthermore, detection and classification of radar signals play an important role in modern radar warning receivers since immediate actions are required in some tasks such as in military applications. Detection of radar signals is extraction of single radar pulses from the environment while classification denotes determining of the modulation category that the pulse produced from. Detecting radar pulses is an important task to take actions against threats in short time. Moreover, intrapulse modulation classification has significance in Electronic Warfare (EW) systems. Electronic support systems which is a subdivision of EW focus on acquiring and intercepting radar signals of possibly threats' to perform analysis of radar features and identity to generate reports. Therefore, intra-pulse modulation classification is important in terms of extracting parameters of threat emitter in decision making.
Various signal processing techniques are proposed for the detection of low probability of intercept (LPI) radar signals. [2] uses Wigner-Ville Hough transform (WVHT) to detect linear frequency modulated LPI radar waveforms. Moreover, [3] employs atomic decomposition to extract time-frequency characteristics of signals. Similarly, [4] investigates signal detection using atomic decomposition with a chirplet dictionary. [5] introduces an algorithm to detect a linear mixture of signals based on atomic decomposition and expectation maximization. [6] discusses a method using multi-channel digital deramping for frequency modulated waves. There are also studies using time-frequency analysis (TFA) techniques for feature extraction to be used in classification. [7] uses short-time Fourier transform (STFT), [8] utilizes Wigner Ville Distribution (WVD) and [9] investigates Choi-William Distribution (CWD). Also, features extracted via autocorrelation function and principal component analysis are used in [10], [11], respectively.

Moreover, many machine learning methods are applied to intra-pulse modulation classification. [12] uses a classifier based on fuzzy Support Vector Machines (SVM) while [13] utilizes mutual information based feature reduction before fuzzy SVM. SVM is also used in [13]. The main problem with mentioned approaches is that features employed may not be explanatory. Therefore, it is not an easy task to determine discriminative features or even extracted features may not be adequate for decent performance. Hence, neural network based approaches become popular for automatic feature extraction. Artificial neural networks are used in [14] while [8] employs convolutional neural networks for feature extraction and classification.

Aforementioned studies focus on either pulse detection or modulation classification. In this study, we present a complete framework performing both detection and classification of radar pulses in the sense of modulation categorization. In the proposed technique, radar signals are assumed to be received in their raw form. In detection phase, we first apply STFT to obtain time-frequency images (TFI) of radar signals. To detect pulses in TFIs, we use the outcomes of Hough transform 
and represent each pulse with a single line. Then, regions of the TFI containing pulses are extracted using these lines. In classification part, we use convolutional neural networks $(\mathrm{CNN})$ to categorize radar pulse signals. At the end, class probabilites are given.

The organization of the paper is as follows. In Section II we provide the problem description while in Section III we investigate pulse detection and intrapulse modulation classification. We illustrate classification results in Section IV. In Section V, we provide certain remarks and conclude the paper.

\section{PROBLEM DESCRIPTION}

We study radar pulse detection and classification in a complete framework where radar signals are assumed to be received as in their raw form within limited time intervals called dwells. Time-frequency images of dwells are obtained using short time Fourier transform. Each dwell corresponds to sequence of radar pulses distributed in time. Moreover, each pulse is associated with a label determining the class that the pulse belongs to. Our goal is to first detect pulses in TFIs and then classify them into a category. Fig. 1 gives complete framework for pulse detection and modulation classification.

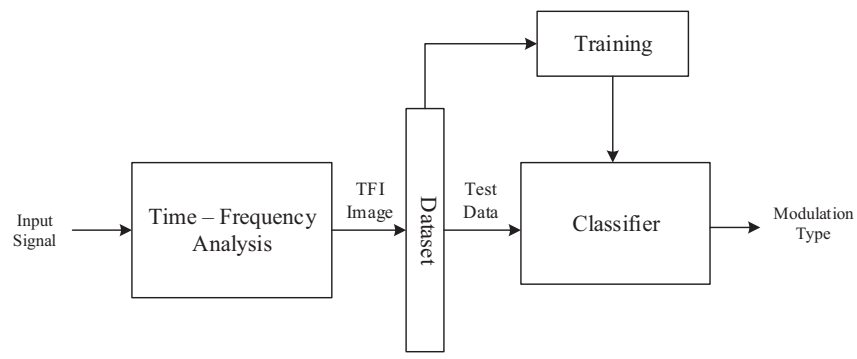

Fig. 1. Proposed complete framework for radar pulse detection and classification.

\section{MEthods}

In this section, we explain the methods used in radar pulse detection and intra-pulse modulation classification.

\section{A. Pulse Detection}

We first use short time Fourier transform to obtain TFI. STFT is obtained by applying the Fourier transform by a fixedsized, moving window to input. STFT is basically segmenting the signal into narrow time intervals in which the signal is assumed to be stationary aand then taking Fourier transform of each segment. Thus, each Fourier transform provides spectral information of different slices of the signal with momentarily time and frequency information. The definition of STFT of a signal $f(t)$ is given by

$$
\operatorname{STFT}\left(t^{\prime}, w\right)=\int_{t}\left[f(t) \cdot W\left(t-t^{\prime}\right)\right] \cdot e^{-j 2 \pi w t}
$$

where $W(t)$ denotes utilized window.
We use Hough transform to detect pulses in TFIs. Hough transform is a method to recognize lines and arbitrary shapes. It represents points in a given image in Hough space. Edge detection is performed first to detect edges in the image. Then, edge points are mapped to the Hough space and the areas where Hough space lines intersect are determined resulting detected lines in the original image. After Hough transform produces a combination of lines for regions expectedly corresponding pulses, we produce a single line for these regions to extract images of pulses. For that purpose, we produce two lines to represent a pulse, one of which is horizontal and the other one is vertical. For a line produced by Hough transform, we first check if there is enough number of lines around it. If this condition is satisfied we say that there is a pulse. Line representing the pulse horizontally are determined using the leftmost and rightmost points of the group of lines of interest. In other words, end points of the horizontal line are determined by the leftmost and rightmost end points of the group of lines. Similarly, we follow the same approach to draw vertical line, i.e., it is produced using the uppermost and lowermost points of the group of lines given by Hough transform. At the end, final line is the longer one of horizontal and vertical lines. To obtain image regions corresponding to pulses, region around the middle point of the final line is extracted.

\section{B. Pulse Classification}

Convolutional neural network is a special type of neural network that makes explicit assumption that the inputs are images. Thus, they are widely used for feature extraction and classification in many tasks related to image processing such as image segmentation, classification and video processing. Their design enables to process images in a more efficient way such that each neuron is connected only a local region of the input volume. In CNNs, input is convolved with neurons and then non-linear operations are performed such as pooling. It is critical to learn the weights of the neurons to capture local similarities in the input. At the end, class probabilites are provided.

In our $\mathrm{CNN}$ structure, we have 3 convolutional layers. The number of filters in each layer is 8,16 and 32 , respectively. We apply batch normalization in each layer after convolution operation. Rectified linear unit is chosen as non-linear function. At the end of each layer but the last one we perform maxpooling of size $2 x 2$ with a stride of 2 to reduce size, thus, decreasing computational load. Fig. 3 shows CNN model we use.

\section{EXPERIMENTS}

In this section, we first describe our dataset and then illustrate the intra-pulse modulation classification results.

\section{A. Dataset}

We construct a dataset of 10 classes for simulations. We consider both frequency and phase modulated radar waveforms 


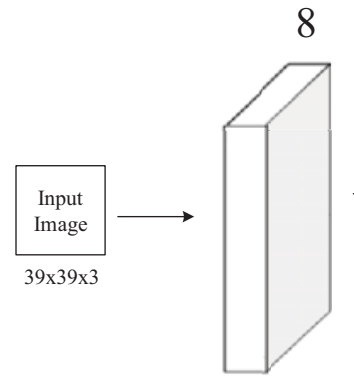

Conv. $3 \times 3$

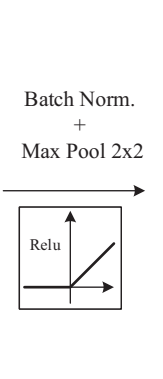

Conv. $3 \times 3$

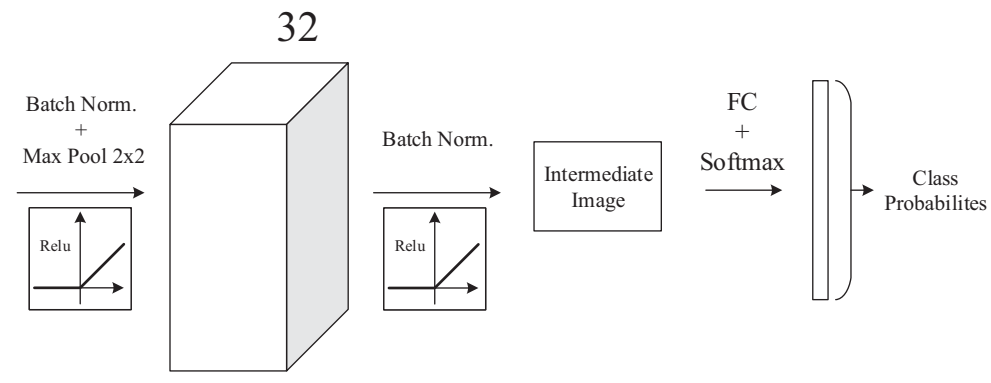

Conv. $3 \times 3$

Fig. 2. Convolutional neural network structure used in complete framework. Model consists of three convolutional layers and class probabilities are provided after softmax. Numbers on convolutional filters denote the number of convolutional filters.
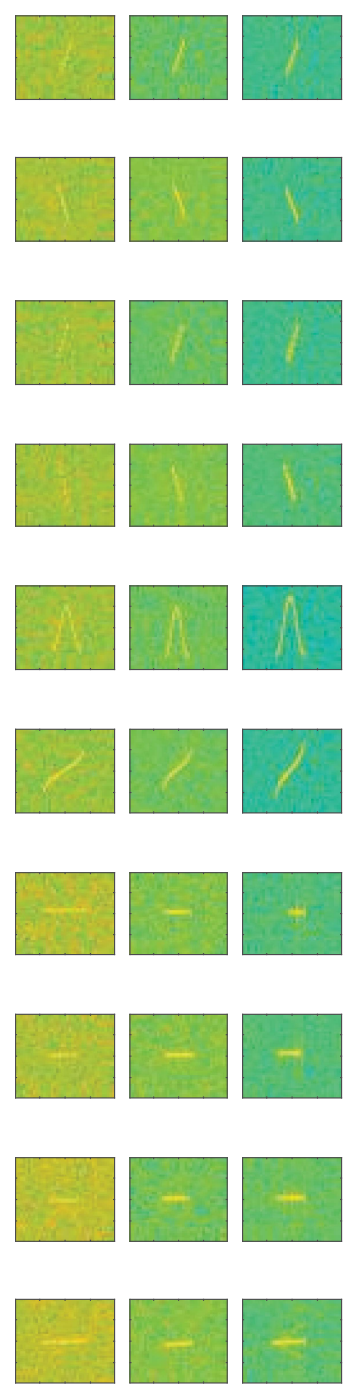

Fig. 3. Examples of all modulations under different SNR. Modulation types are in the order given in Table I from up to bottom while SNR values are $-10,0$ and $10 \mathrm{~dB}$ from left to right.

in supervised learning framework. The modulation types con-
TABLE I

MODULATION TYPES

\begin{tabular}{c|c} 
Frequency Modulation (FM) & Phase Modulation (PM) \\
\hline Sawtooth Upchirp & Barker \\
Sawtooth Downchirp & Hadamard \\
Stepped Upchirp & P1 \\
Stepped Downchirp & T1 \\
Sinusoidal & \\
NFLM-Taylor &
\end{tabular}

sidered in the simulations are given in Table I.

We consider different SNR levels in simulations which are $-10 \mathrm{~dB}, 0 \mathrm{~dB}$ and $10 \mathrm{~dB}$. Thus, a different set is produced for each SNR. To compose a dataset with various samples of distinct modulation types, we consider different pulse width values for both FM and PM modulated waveforms as well as different bandwidths for FM modulated waveforms. Specifically, we use the set for pulse width $\{1,1.5,2,2.5,3,3.5,4,4.5,5\}$ $\mu$ s and $100-200 \mathrm{MHz}$ interval with $10 \mathrm{MHz}$ increments for bandwidth of radar pulses. Moreover, the number of steps in stepped modulated waves is 5 while taylor number is 5 for NFLM-Taylor waveform. Code number for all PM modulated waves but Barker is 4 whereas phase change is 13 for $\mathrm{T} 1$ modulation. Code number for Barker is taken as 2. Pulse repetition intervals for all modulations are constant and set to $10 \mu \mathrm{s}$. All other parameters for modulated radar pulses are same. Sampling frequency is $2500 \mathrm{MHz}$ and window length used while producing TFIs is chosen as 512. Fig. 3 demonstrates examples of all modulation types under different SNR values. Images correspond to FM and PM modulated waves with the order given in Table I from up to bottom while SNR values used to produce dataset are $-10,0$ and $10 \mathrm{~dB}$ from left to right.

\section{B. Simulations}

In this part, we give classification results. We use accuracy value as performance measure. It is the percentage of correctly classified samples to the number of all samples in the dataset. 
TABLE II

CONFUSION MATRIX FOR -10 DB SNR

\begin{tabular}{|c|c|c|c|c|c|c|c|c|c|c|}
\hline $\begin{array}{ll}\text { True } & \text { Predicted }\end{array}$ & Sawtooth Up & Sawtooth Down & Stepped Up & Stepped Down & Sin & NFLM-Taylor & Barker & Hadamard & $\mathrm{P} 1$ & $\mathrm{~T} 1$ \\
\hline Sawtooth Up & 121 & 0 & 0 & 0 & 1 & 27 & 0 & 0 & 0 & 1 \\
\hline Sawtooth Down & 0 & 147 & 0 & 1 & 2 & 0 & 0 & 0 & 0 & 0 \\
\hline Stepped Up & 1 & 0 & 147 & 1 & 0 & 1 & 0 & 0 & 0 & 0 \\
\hline Stepped Down & 0 & 0 & 2 & 147 & 1 & 0 & 0 & 0 & 0 & 0 \\
\hline Sin & 3 & 1 & 0 & 0 & 143 & 2 & 1 & 0 & 0 & 0 \\
\hline NFLM-Taylor & 9 & 0 & 0 & 1 & 3 & 137 & 0 & 0 & 0 & 0 \\
\hline Barker & 0 & 0 & 0 & 1 & 0 & 0 & 42 & 48 & 40 & 19 \\
\hline Hadamard & 0 & 0 & 0 & 0 & 0 & 0 & 28 & 48 & 51 & 23 \\
\hline $\mathrm{P} 1$ & 0 & 1 & 0 & 0 & 0 & 0 & 33 & 45 & 44 & 27 \\
\hline $\mathrm{T} 1$ & 0 & 0 & 0 & 0 & 0 & 1 & 33 & 32 & 36 & 48 \\
\hline
\end{tabular}

TABLE III

CONFUSION MATRIX FOR 0 DB SNR

\begin{tabular}{|c|c|c|c|c|c|c|c|c|c|c|}
\hline True $\quad$ Predicted & Sawtooth Up & Sawtooth Down & Stepped Up & Stepped Down & Sin & NFLM-Taylor & Barker & Hadamard & $\mathrm{P} 1$ & $\mathrm{~T} 1$ \\
\hline Sawtooth Up & 143 & 0 & 1 & 0 & 1 & 5 & 0 & 0 & 0 & 0 \\
\hline Sawtooth Down & 0 & 148 & 0 & 1 & 1 & 0 & 0 & 0 & 0 & 0 \\
\hline Stepped Up & 0 & 0 & 148 & 2 & 0 & 0 & 0 & 0 & 0 & 0 \\
\hline Stepped Down & 0 & 0 & 0 & 150 & 0 & 0 & 0 & 0 & 0 & 0 \\
\hline Sin & 0 & 0 & 0 & 1 & 149 & 0 & 0 & 0 & 0 & 0 \\
\hline NFLM-Taylor & 6 & 0 & 2 & 0 & 0 & 142 & 0 & 0 & 0 & 0 \\
\hline Barker & 0 & 0 & 0 & 0 & 0 & 0 & 63 & 45 & 39 & 3 \\
\hline Hadamard & 0 & 0 & 0 & 0 & 0 & 0 & 16 & 103 & 30 & 1 \\
\hline P1 & 0 & 0 & 0 & 0 & 0 & 0 & 27 & 39 & 78 & 5 \\
\hline T1 & 0 & 0 & 0 & 0 & 0 & 0 & 15 & 17 & 21 & 97 \\
\hline
\end{tabular}

TABLE IV

CONFUSION MATRIX FOR 10 DB SNR

\begin{tabular}{|c|c|c|c|c|c|c|c|c|c|c|}
\hline True $\quad$ Predicted & Sawtooth Up & Sawtooth Down & Stepped Up & Stepped Down & Sin & NFLM-Taylor & Barker & Hadamard & $\mathrm{P} 1$ & $\mathrm{~T} 1$ \\
\hline Sawtooth Up & 142 & 0 & 0 & 1 & 1 & 5 & 0 & 1 & 0 & 0 \\
\hline Sawtooth Down & 0 & 150 & 0 & 0 & 0 & 0 & 0 & 0 & 0 & 0 \\
\hline Stepped Up & 0 & 0 & 150 & 0 & 0 & 0 & 0 & 0 & 0 & 0 \\
\hline Stepped Down & 0 & 2 & 0 & 148 & 0 & 0 & 0 & 0 & 0 & 0 \\
\hline Sin & 0 & 0 & 0 & 0 & 150 & 0 & 0 & 0 & 0 & 0 \\
\hline NFLM-Taylor & 2 & 0 & 0 & 0 & 1 & 147 & 0 & 0 & 0 & 0 \\
\hline Barker & 0 & 1 & 0 & 0 & 0 & 0 & 115 & 13 & 17 & 4 \\
\hline Hadamard & 0 & 0 & 0 & 0 & 0 & 0 & 16 & 129 & 5 & 0 \\
\hline P1 & 0 & 0 & 0 & 0 & 0 & 0 & 11 & 10 & 129 & 0 \\
\hline T1 & 0 & 0 & 0 & 0 & 0 & 0 & 4 & 0 & 0 & 146 \\
\hline
\end{tabular}

We train a separate classifier for each SNR value and obtain performance results using test sets that the classifiers have never seen. Moreover, we perform tests for the classifiers that are trained in each SNR value using test sets of different SNR values, i.e., we train a classifier in $-10 \mathrm{~dB}$ SNR and conduct tests using 0 and $10 \mathrm{~dB}$ SNRs. Since the environment conditions may alter SNR may be different. Thus, a classifier trained in a specific SNR value may be employed under different SNRs. We test classifiers trained under different SNRs to see the effect this type of a change.

We use 650 training, 100 validation and 150 test samples for each modulation type when training and test are conducted in same SNR values. When training and test are performed for different SNR values whole sets are used for training and test in respective SNRs. In this case, sets consist of 900 samples. Tables II, III and IV give the confusion matrices for $-10 \mathrm{~dB}$, $0 \mathrm{~dB}$ and $10 \mathrm{~dB}$ SNR, respectively. Results demonstrate that as SNR value increases classification performance improves. This outcome is expected since radar pulses is much more visible in high SNR. Classification performance is much better for frequency modulated pulses than phase modulated pulses. Since frequency does not change visibly for phase modulated pulses it is harder to distinguish PM pulses. Therefore, in high SNR case details for PM pulses are more clear yielding better classification performance in this case.

Classification of frequency modulated pulses yields good performance even for low SNR value. For FM pulses, sawtooth upchirp and NFLM-Taylor are confused. Other than that, the 
TABLE V

ClassifiCATION ACCURACIES UNDER DIFFERENT SNR IN TRAIN AND TEST

\begin{tabular}{c|c|c} 
Train & Test & Accuracy \\
\hline$-10 \mathrm{~dB}$ & $-10 \mathrm{~dB}$ & $\mathbf{6 8 . 2 7} \%$ \\
$-10 \mathrm{~dB}$ & $0 \mathrm{~dB}$ & $65.49 \%$ \\
$-10 \mathrm{~dB}$ & $10 \mathrm{~dB}$ & $59.44 \%$ \\
$0 \mathrm{~dB}$ & $-10 \mathrm{~dB}$ & $38.58 \%$ \\
$0 \mathrm{~dB}$ & $0 \mathrm{~dB}$ & $\mathbf{8 1 . 4 0} \%$ \\
$0 \mathrm{~dB}$ & $10 \mathrm{~dB}$ & $77.57 \%$ \\
$10 \mathrm{~dB}$ & $-10 \mathrm{~dB}$ & $15.67 \%$ \\
$10 \mathrm{~dB}$ & $0 \mathrm{~dB}$ & $64.17 \%$ \\
$10 \mathrm{~dB}$ & $10 \mathrm{~dB}$ & $\mathbf{9 3 . 7 3} \%$
\end{tabular}

other modulations are distinguished clearly. For PM pulses, radar pulses are not completely separated to distinct classes even for high SNR case. Moreover, Table I provides classification accuracies trained and tested in all combinations of SNR values. As expected, when SNR increases accuracy improves for same train and test SNR. In other cases, classification performance decreases significantly. The performance reduction is much less when SNR increases. This behavior can be seen when SNR increases both from $-10 \mathrm{~dB}$ to $0 \mathrm{~dB}$ and from $0 \mathrm{~dB}$ to $10 \mathrm{~dB}$. On the other hand, the opposite of this behavior is observed in reverse case, i.e., when SNR decreases for example from $10 \mathrm{~dB}$ to $0 \mathrm{~dB}$. In these cases, performance reduces severely such that trained classifier is not suitable to use.

\section{CONCLUSION}

We study radar pulse detection and modulation classification where radar pulses are assumed to be received in their raw form. In this manner, we provide a complete framework performing both detection and classification. Raw radar signals are processed to obtain TFIs using STFT. Then, we employ Hough transform to detect radar pulses in TFIs and denote these pulses with a single line. Convolutional neural networks are used to perform intra-pulse modulation of radar signals. Classification performance is given with respect to different SNR values in terms of accuracy.

\section{REFERENCES}

[1] S. Ayazgok, C. Erdem, M. T. Ozturk, A. Orduyilmaz, and M. Serin, "Automatic antenna scan type classification for next-generation electronic warfare receivers," IET Radar, Sonar Navigation, vol. 12, no. 4 pp. $466-474,2018$

[2] F. G. Geroleo and M. Brandt-Pearce, "Detection and estimation of lfmcw radar signals," IEEE Transactions on Aerospace and Electronic Systems, vol. 48, no. 1, pp. 405-418, Jan 2012.

[3] G. Lopez-Risueno, J. Grajal, and O. Yeste-Ojeda, "Atomic decomposition-based radar complex signal interception," IEE Proceedings - Radar, Sonar and Navigation, vol. 150, no. 4, pp 323-, Aug 2003.

[4] G. Lopez-Risueno and J. Grajal, "Unknown signal detection via atomic decomposition," in Proceedings of the 11th IEEE Signal Processing Workshop on Statistical Signal Processing (Cat. No.01TH8563), Aug 2001, pp. 174-177.
[5] G. Lopez Risueno and J. Grajal, "Multiple signal detection and estimation using atomic decomposition and em," IEEE Transactions on Aerospace and Electronic Systems, vol. 42, no. 1, pp. 84-102, Jan 2006.

[6] S. Jie, T. Xiao-ming, and H. You, "Muti-channel digital lpi signal detector," in 2006 CIE International Conference on Radar, Oct 2006 pp. $1-4$.

[7] G. Lopez-Risueno, J. Grajal, and A. Sanz-Osorio, "Digital channelized receiver based on time-frequency analysis for signal interception," IEEE Transactions on Aerospace and Electronic Systems, vol. 41, no. 3, pp. 879-898, July 2005.

[8] C. Wang, J. Wang, and X. Zhang, "Automatic radar waveform recognition based on time-frequency analysis and convolutional neural network," in 2017 IEEE International Conference on Acoustics, Speech and Signal Processing (ICASSP), March 2017, pp. 2437-2441.

[9] M. Zhang, M. Diao, and L. Guo, "Convolutional neural networks for automatic cognitive radio waveform recognition," IEEE Access, vol. 5, pp. $11074-11082,2017$.

[10] and H. Gao and, "Radar signal classification based on auto-correlation function and directed graphical model," in 2016 IEEE International Conference on Signal Processing, Communications and Computing (ICSPCC), Aug 2016, pp. 1-4.

[11] Z. Yu, C. Chen, and W. Jin, "Radar signal automatic classification based on pca," in 2009 WRI Global Congress on Intelligent Systems, vol. 3, May 2009, pp. 216-220.

[12] R. Mingqiu, C. Jinyan, and Z. Yuanqing, "Classification of radar signals using time-frequency transforms and fuzzy clustering," 052010.

[13] and and and, "Radar emitter signal classification based on mutual information and fuzzy support vector machines," in 2008 9th International Conference on Signal Processing, Oct 2008, pp. 1641-1646.

[14] J. Lunden and V. Koivunen, "Automatic radar waveform recognition," IEEE Journal of Selected Topics in Signal Processing, vol. 1, no. 1, pp. 124-136, June 2007. 